\title{
The Use of Thiazoles in the Halogen Dance Reaction: Application to the Total Synthesis of WS75624 B
}

\section{Supporting Info}

\author{
Eric L. Stangeland and Tarek Sammakia,* \\ Department of Chemistry and Biochemistry, University of Colorado \\ Boulder, Colorado 80309-0215
}

General: All moisture sensitive reactions were conducted under a nitrogen atmosphere in oven-dried glassware using solvents purified according to standard procedures. ${ }^{1}{ }^{1} \mathrm{H}$ NMR spectra were obtained at $500 \mathrm{MHz},{ }^{13} \mathrm{C}$ NMR spectra at $125 \mathrm{MHz}$ with ${ }^{1} \mathrm{H}$ decoupling (WALTZ) in chloroform- $d$, with chemical shifts reported in parts per million referenced to residual chloroform $(\delta$ $=7.24$ for ${ }^{1} \mathrm{H}$ and 77.00 for ${ }^{13} \mathrm{C}$ ). Infrared spectra were recorded as thin films on $\mathrm{NaCl}$ plates. Melting points are uncorrected.

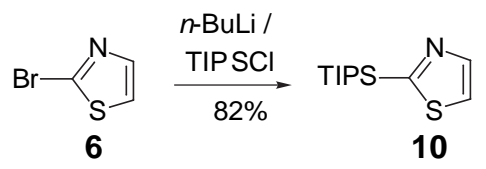

2-Triisopropylsilylthiazole (10). 2-Bromothiazole (6, $492 \mathrm{mg}, 3.0 \times 10^{-3}$ mol, 1 equiv) was added dropwise to $n$-BuLi $\left(2.26 \mathrm{~mL}, 1.46 \mathrm{M}\right.$ in hexanes, 1.1 equiv) in THF $(8 \mathrm{~mL})$ at $-78^{\circ} \mathrm{C}$. The solution was allowed to stir for 1 hour, and then triisopropylsilyl triflate $\left(1.29 \mathrm{~mL}, 3.9 \times 10^{-3} \mathrm{~mol}\right.$, 1.3 equiv) was added dropwise over 1 minute. The solution was stirred at $-78{ }^{\circ} \mathrm{C}$ for 1 hour, and allowed to come to room temperature. The reaction was diluted with ethyl acetate, and washed with saturated $\mathrm{NaHCO}_{3}$, and brine, dried over $\mathrm{MgSO}_{4}$, and concentrated under reduced pressure. Purification by flash chromatography $\left(\mathrm{SiO}_{2}, 2 / 1\right.$ hexanes/chloroform) provided 2triisopropylthiazole (10) (480 mg, 66\%); $R f=0.5$ (10/1 hexanes/ethyl acetate); IR: 2945, 2867, $1463 \mathrm{~cm}^{-1} ;{ }^{1} \mathrm{H} \mathrm{NMR}\left(\mathrm{CDCl}_{3}, 500 \mathrm{MHz}\right) \delta 1.12(\mathrm{~d}, J=7.4 \mathrm{~Hz}, 18 \mathrm{H}), 1.44$ (sept, $\left.J=7.4 \mathrm{~Hz}, 1 \mathrm{H}\right)$, $7.52(\mathrm{~d}, J=3.0 \mathrm{~Hz}, 1 \mathrm{H}), 8.14(\mathrm{~d}, J=3.0 \mathrm{~Hz}, 1 \mathrm{H}) ;{ }^{13} \mathrm{C} \mathrm{NMR}\left(\mathrm{CDCl}_{3}, 125 \mathrm{MHz}\right) \delta 11.68,18.45$, 120.96, 145.38, 169.90. Anal. Calcd for $\mathrm{C}_{12} \mathrm{H}_{23} \mathrm{NSSi}$ C, 59.69; H, 9.60; N, 5.80; S, 13.28. Found C, 59.73; H, 9.86; N, 5.68; S, 13.68.

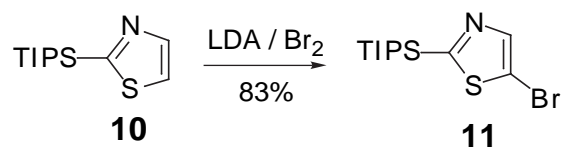

5-Bromo-2-triisopropylsilyl Thiazole (11). $n$-BuLi $(0.71 \mathrm{~mL}, 1.60 \mathrm{M}$ in hexanes, 1.6 equiv) was added dropwise to 2-triisopropylsilyl thiazole (10, $170 \mathrm{mg}, 7.0 \mathrm{x} 10-4 \mathrm{~mol}, 1$ equiv) in THF (8 $\mathrm{mL})$ at $-78^{\circ} \mathrm{C}$. The solution was allowed to stir for 2 hours, and then bromine $\left(108 \mu \mathrm{L}, 2.1 \times 10^{-3}\right.$ mol, 3 equiv) was added dropwise over 1 minute. The solution was stirred at $-78^{\circ} \mathrm{C}$ for 1 hour, and then allowed to come to room temperature. The reaction was diluted with ethyl acetate, and washed with saturated $\mathrm{NaHCO}_{3}$, and brine, dried over $\mathrm{MgSO}_{4}$, and concentrated under reduced pressure. Purification by flash chromatography $\left(\mathrm{SiO}_{2}\right.$, hexanes then 100/1 hexanes/ethyl acetate) provided 5bromo-2-triisopropylthiazole (11) (187 mg, 83\%); $R f=0.8$ (50/1 hexanes/ethyl acetate); IR: 2949, 2867, 1462, $1444 \mathrm{~cm}^{-1} ;{ }^{1} \mathrm{H}$ NMR $\left(\mathrm{CDCl}_{3}, 500 \mathrm{MHz}\right) \delta 1.10(\mathrm{~d}, J=7.4 \mathrm{~Hz}, 18 \mathrm{H}), 1.39$ (sept, $J=$ $7.4 \mathrm{~Hz}, 1 \mathrm{H}), 7.95(\mathrm{~s}, 1 \mathrm{H}) ;{ }^{13} \mathrm{C} \mathrm{NMR}\left(\mathrm{CDCl}_{3}, 125 \mathrm{MHz}\right) \delta 11.54,18.39,111.59,146.49,173.44$. Anal. Calcd for $\mathrm{C}_{12} \mathrm{H}_{22}$ BrNSSi C, 44.99; H, 6.92; N, 4.37. Found C, 44.94; H, 7.10; N, 4.28. 


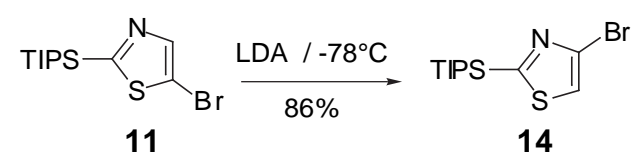

4-Bromo-2-Triisopropylsilylthiazole (14). 5-Bromo-2-triisopropylsilyl thiazole $(\mathbf{1 1}, 77 \mathrm{mg}$, $2.4 \times 10^{-4}$ mol, 1 equiv) in THF (3 mL) was added dropwise to a solution of LDA in THF (5 mL) prepared from diisopropylamine $\left(50 \mathrm{~mL}, 3.6 \times 10^{-4} \mathrm{~mol}, 1.5\right.$ equiv) and $n$-BuLi $(0.18 \mathrm{~mL}, 1.6 \mathrm{M}$ in hexanes, 1.2 equiv) at $-78{ }^{\circ} \mathrm{C}$. The solution was allowed to stir for 3 hours, and then quenched with water. The reaction was diluted with ethyl acetate, and the organic layer was washed with saturated $\mathrm{NaHCO}_{3}$, and brine, dried over $\mathrm{MgSO}_{4}$, and concentrated under reduced pressure. Purification by flash chromatography ( $\mathrm{SiO} 2$, hexanes then 100/1 hexanes/ethyl acetate) provided 4-bromo-2triisopropylthiazole (14) (66 mg, 86\%); $R f=0.9$ (50/1 hexanes/ethyl acetate); IR (neat): 2945, 2867, $1462,1443 \mathrm{~cm}^{-1} ;{ }^{1} \mathrm{H} \mathrm{NMR}\left(\mathrm{CDCl}_{3}, 500 \mathrm{MHz}\right) \delta 1.11(\mathrm{~d}, J=7.4 \mathrm{~Hz}, 18 \mathrm{H}), 1.43$ (sept, $J=7.4 \mathrm{~Hz}$, $1 \mathrm{H}), 7.38(\mathrm{~s}, 1 \mathrm{H}) ;{ }^{13} \mathrm{C} \mathrm{NMR}\left(\mathrm{CDCl}_{3}, 125 \mathrm{MHz}\right) \delta 11.54,18.40,119.05,127.96,172.05$. Anal. Calcd for $\mathrm{C}_{12} \mathrm{H}_{22} \mathrm{BrNSSi}$ C, 44.99; H, 6.92; N, 4.37; S, 10.01. Found C, 45.28; H, 7.18; N, 3.97; S, 10.01 .

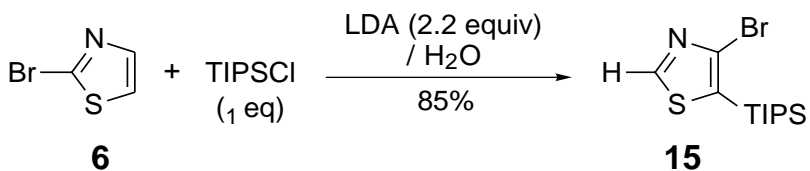

4-Bromo-5-triisopropylsilylthiazole (15). $n$-BuLi $(0.85 \mathrm{~mL}, 1.58 \mathrm{M}$ in hexanes, 2.2 equiv) was added dropwise to diisopropylamine $\left(0.21 \mathrm{~mL}, 1.46 \times 10^{-3} \mathrm{~mol}, 2.4\right.$ equiv) in THF $(8 \mathrm{~mL})$ at $0{ }^{\circ} \mathrm{C}$. After stirring for 15 minutes, the solution was cooled to $-78{ }^{\circ} \mathrm{C}$. Triisopropylsilyl chloride $(0.14$ $\mathrm{mL}, 6.7 \times 10^{-4} \mathrm{~mol}, 1.1$ equiv) was added to the vigorously stirring LDA solution via syringe followed by 2-bromothiazole $\left(6,55 \mu \mathrm{L}, 6.1 \times 10^{-4} \mathrm{~mol}, 1\right.$ equiv) via syringe over 5 seconds. The light yellow solution was stirred for 2 hours at $-78^{\circ} \mathrm{C}$. The reaction was then quenched with water at $-78{ }^{\circ} \mathrm{C}$, and allowed to come to room temperature. The reaction was diluted with ethyl acetate, and the organic layer was washed with $1 \mathrm{M} \mathrm{NH}_{4} \mathrm{OH}$, saturated $\mathrm{NaHCO}_{3}$, and brine, dried over $\mathrm{MgSO}_{4}$, and concentrated under reduced pressure. Purification by flash chromatography $\left(\mathrm{SiO}_{2}\right.$, 100/1 hexanes/diethyl ether) provided 4-bromo-5-triisopropylsilyl thiazole (15) (166 mg, 85\% yield). $R f=0.6$ (10:1 hexanes/ethyl acetate); IR (neat): 2942, $1434 \mathrm{~cm}^{-1} ;{ }^{1} \mathrm{H} \mathrm{NMR}\left(\mathrm{CDCl}_{3}, 500\right.$ $\mathrm{MHz}) \delta 1.11(\mathrm{~d}, J=7.6 \mathrm{~Hz}, 18 \mathrm{H}), 1.56(\mathrm{sept}, J=7.6 \mathrm{~Hz}, 1 \mathrm{H}), 8.88(\mathrm{~s}, 1 \mathrm{H}) ;{ }^{3} \mathrm{C} \mathrm{NMR}\left(\mathrm{CDCl}_{3}\right.$, $125 \mathrm{MHz}) \mathrm{d} 12.34,18.59,124.39,133.15,157.52$.

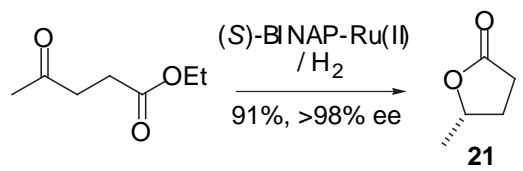

(S)-4-Methyl- $\gamma$-butyrolactone (21). ${ }^{2}$ Ethyl levulinate (14.84 g, 0.103 mol, 1.0 equiv), ethanol $(15 \mathrm{~mL})$, and $\mathrm{HCl}(512 \mu \mathrm{L}, 1.0 \mathrm{M}$ aqueous, $0.5 \%)$ were degassed with nitrogen for 1 hour then transferred to a flask containing $\mathrm{RuCl}_{2}-(S)$-BINAP ${ }^{-E_{3}}{ }_{3} \mathrm{~N}\left(46 \mathrm{mg}, 5.1 \times 10^{-5} \mathrm{~mol}, 0.05 \%\right)$. After the catalyst had dissolved, the solution was transferred via cannula under nitrogen atmosphere to a stainless steel bomb reactor equipped with a glass insert. The reactor was purged $3 \mathrm{x}$ with hydrogen, and placed under 1400 psi hydrogen pressure and brought to $50{ }^{\circ} \mathrm{C}$ for 5 days, with occasional recharging of hydrogen pressure. After 5 days, the pressure was released and the reaction was concentrated to an oil. Kugelrhor distillation $\left(48{ }^{\circ} \mathrm{C}, 0.5 \mathrm{mmHg}\right)$ provided $(S)-4-$ methyl- $\gamma$-butyrolactone (21) $\left(9.38 \mathrm{~g}, 9.37 \times 10^{-2} \mathrm{~mol}, 91 \%\right.$ yield, $>98 \%$ ee by GC using Supleco $\beta$ dex column). $[\alpha]_{\mathrm{D}}-35.03^{\circ}$ (c $15.3 \mathrm{mg} / \mathrm{mL}, \mathrm{CH}_{2} \mathrm{Cl}_{2}$ ); IR (neat): 2978, 1774, $1197 \mathrm{~cm}^{-1}$; ${ }^{1} \mathrm{H} \mathrm{NMR}$ $\left(\mathrm{CDCl}_{3}, 500 \mathrm{MHz}\right) \delta 1.38(\mathrm{~d}, J=6.2 \mathrm{~Hz}, 3 \mathrm{H}), 1.80(\mathrm{~m}, 1 \mathrm{H}), 2.33(\mathrm{~m}, 1 \mathrm{H}), 2.52(\mathrm{~m}, 2 \mathrm{H}), 4.61(\mathrm{~m}$, $1 \mathrm{H}) ;{ }^{13} \mathrm{C} \mathrm{NMR}\left(\mathrm{CDCl}_{3}, 125 \mathrm{MHz}\right) \delta 21.04,29.06,29.69,77.21,177.17$. 


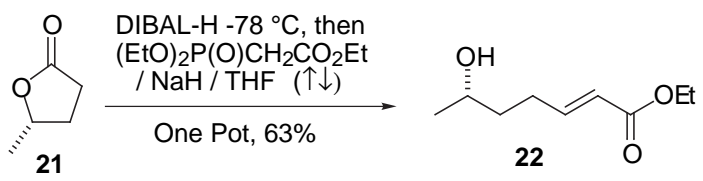

(S)-Ethyl-6-hydroxy-hept-2-enoate (22). (S)-4-Methyl- $\gamma$-butyrolactone $(\mathbf{2 1})(6.0 \mathrm{~g}, 6.0 \mathrm{x} \mathrm{10-2}$ mol, 1.0 equiv) was dissolved in THF $(240 \mathrm{~mL})$ under a nitrogen atmosphere and cooled to $-78{ }^{\circ} \mathrm{C}$. Diisobutylaluminum hydride $(63 \mathrm{~mL}, 1.0 \mathrm{M}$ in hexanes, 1.05 equiv) was added dropwise over a period of 20 minutes, and the reaction was stirred an additional hour. To a separate flask containing a slurry of sodium hydride $\left(2.87 \mathrm{~g}, 1.2 \times 10^{-1} \mathrm{~mol}, 2.0\right.$ equiv) in THF (400 mL) under a nitrogen atmosphere, triethyl phosphonoactetate $\left(13.08 \mathrm{~mL}, 6.6 \times 10^{-2} \mathrm{~mol}, 1.2\right.$ equiv) was added dropwise over a period of 1 hour with vigorous $\mathrm{H}_{2}$ evolution. This mixture was then transferred to the reaction flask via cannula over a period of 30 minutes. The reaction was allowed to come to room temperature and stirred for 18 hours or until the lactol was no longer observed by TLC (lactol: $R f=$ 0.5 , UV, product: $R f=0.6$, UV; $3: 2$ ethyl acetate/hexanes). The reaction was diluted with hexanes $(300 \mathrm{~mL})$ and $\mathrm{NaOH}(3 \mathrm{~mL}, 10 \mathrm{M})$ was added dropwise by pipet. The reaction was allowed to stir at room temperature for 1 hour at which time a large amount of aluminum salts had precipitated. The reaction mixture was dried with $\mathrm{MgSO}_{4}$, filtered and washed with hexanes, and the filtrate concentrated under reduced pressure. Flash chromatography $\left(\mathrm{SiO}_{2}, 2 / 1\right.$ hexanes/ ethyl acetate) provied $(S)$-ethyl-6-hydroxy-hept-2-enoate (22) (6.59 g, 63\%). Rf 0.6 (4:6 hexanes/ ethyl acetate); $[\alpha]_{\mathrm{D}}+13.17^{\circ}\left(\mathrm{c} 12.0 \mathrm{mg} / \mathrm{mL}, \mathrm{CH}_{2} \mathrm{Cl}_{2}\right.$ ); IR (neat): 3436, 2970, 1718, 1653, 1204, $1046 \mathrm{~cm}^{-1} ;{ }^{1} \mathrm{H}$ NMR $\left(\mathrm{CDCl}_{3}, 500 \mathrm{MHz}\right) \delta 1.19(\mathrm{~d}, J=6.2 \mathrm{~Hz}, 3 \mathrm{H}), 1.26(\mathrm{t}, J=7.1 \mathrm{~Hz}, 3 \mathrm{H}), 1.42(\mathrm{bs}, 1 \mathrm{H}), 1.58$ $(\mathrm{m}, 2 \mathrm{H}), 2.29(\mathrm{~m}, 2 \mathrm{H}), 3.80$ (sextet, $J=6.3 \mathrm{~Hz}, 1 \mathrm{H}), 4.15(\mathrm{q}, J=7.1 \mathrm{~Hz}, 2 \mathrm{H}), 5.81(\mathrm{~d}, J=15.6 \mathrm{~Hz}$, $1 \mathrm{H}), 6.95(\mathrm{dt}, J=6.9,15.6 \mathrm{~Hz}, 1 \mathrm{H}) ;{ }^{13} \mathrm{C} \mathrm{NMR}\left(\mathrm{CDCl}_{3}, 125 \mathrm{MHz}\right) \delta 14.24,23.62,28.46,37.25$, 60.18, 67.26, 121.55, 148.64, 166.63. Anal. Calcd for $\mathrm{C}_{9} \mathrm{H}_{16} \mathrm{O}_{3} \mathrm{C}, 62.77 ; \mathrm{H}, 9.36$. Found: C, $63.13 ; \mathrm{H}, 9.76$

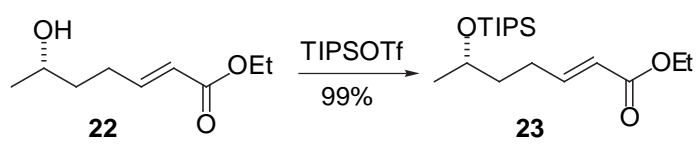

(S)-Ethyl-6-(triisopropylsiloxy)-hept-2-enoate (23). (S)-Ethyl-6-hydroxy-hept-2-enoate (22, $6.59 \mathrm{~g}, 3.82 \times 10^{-2}$ mol, 1.0 equiv) and ethyl diisopropyl amine $\left(13.3 \mathrm{~mL}, 7.64 \times 10^{-2} \mathrm{~mol}, 2.0\right.$ equiv) were dissolved in THF $(200 \mathrm{~mL})$. Triisopropylsilyl triflate $\left(10.79 \mathrm{~mL}, 4.01 \times 10^{-3} \mathrm{~mol}\right.$, 1.05 equiv) was added dropwise over a period of 1 minute, and then stirred an additional hour. The reaction was quenched with water and diluted with ethyl acetate. The organic layer was washed with $1 \mathrm{M} \mathrm{HCl}$, saturated $\mathrm{NaHCO}_{3}$, and brine, dried over $\mathrm{MgSO}_{4}$, and concentrated under reduced pressure. Flash chromatography $\left(\mathrm{SiO}_{2}, 3 / 1\right.$ hexanes/ethyl acetate) provided $(S)$-ethyl-6(triisopropylsiloxy)-hept-2-enoate $(\mathbf{2 3})(12.43 \mathrm{~g}, 99 \%)$. Rf 0.6 (4:6 hexanes/ ethyl acetate); $[\alpha]_{\mathrm{D}}$ $+5.83^{\circ}$ (c $9.6 \mathrm{mg} / \mathrm{mL}, \mathrm{CH}_{2} \mathrm{Cl}_{2}$ ); IR (neat): 2942, 1724, 1655, 1463, 1205, $1045 \mathrm{~cm}^{-1}$; ${ }^{1} \mathrm{H} \mathrm{NMR}$ $\left(\mathrm{CDCl}_{3}, 500 \mathrm{MHz}\right) \delta 1.03(\mathrm{~s}, 21 \mathrm{H}), 1.15(\mathrm{~d}, J=6.2 \mathrm{~Hz}, 3 \mathrm{H}), 1.26(\mathrm{t}, J=7.1 \mathrm{~Hz}, 3 \mathrm{H}), 1.59(\mathrm{~m}, 2 \mathrm{H})$, $2.25(\mathrm{q}, J=7.0 \mathrm{~Hz}, 2 \mathrm{H}), 3.97$ (sextet, $J=6.0 \mathrm{~Hz}, 2 \mathrm{H}), 4.16(\mathrm{q}, J=7.1 \mathrm{~Hz}, 2 \mathrm{H}), 5.79(\mathrm{~d}, J=15.6$ $\mathrm{Hz}, 1 \mathrm{H}), 6.97(\mathrm{dt}, J=6.8,15.7 \mathrm{~Hz}, 1 \mathrm{H}) ;{ }^{13} \mathrm{C} \mathrm{NMR}\left(\mathrm{CDCl}_{3}, 125 \mathrm{MHz}\right) \delta 12.48,14.28,18.12$, 23.36, 27.87, 37.92, 60.13, 67.74, 121.16, 149.39, 166.73; Anal. Calcd for $\mathrm{C}_{18} \mathrm{H}_{36} \mathrm{O}_{3} \mathrm{Si}$; C, 65.80; H, 11.04. Found: C, 65.68; H, 10.82 .

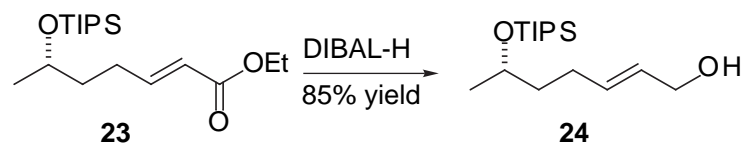

(S)-6-(triisopropylsiloxy)-hept-2-en-1-ol (24). Diisobutylaluminum hydride (25.3 mL, 1.0 M in hexanes, 3.0 equiv) was added dropwise over a period of 10 minutes to a solution of (S)-ethyl-6(triisopropylsiloxy)-hept-2-enoate $\left(\mathbf{2 3}, 2.77 \mathrm{~g}, 8.42 \times 10^{-3} \mathrm{~mol}, 1.0\right.$ equiv) in THF $(50 \mathrm{~mL})$ at $0{ }^{\circ} \mathrm{C}$, and then stirred for 1 hour. The reaction was quenched with water, stirred 1 hour, and then diluted with ethyl acetate. The organic layer was washed with $1 \mathrm{M} \mathrm{HCl}$, saturated $\mathrm{NaHCO}_{3}$, and brine, dried over $\mathrm{MgSO}_{4}$, and concentrated under reduced pressure. Flash chromatography $\left(\mathrm{SiO}_{2}, 10 / 1\right.$ 
hexanes/ethyl acetate) provided (S)-ethyl-6-(triisopropylsiloxy)-hept-2-enol (24) (2.06 g, 85\%). $R f$ 0.2 (10:1 hexanes/ethyl acetate); $[\alpha]_{\mathrm{D}}+5.00^{\circ}\left(\mathrm{c} 8.3 \mathrm{mg} / \mathrm{mL}, \mathrm{CH}_{2} \mathrm{Cl}_{2}\right)$; IR (neat): $3323,2962,1463$, $1012 \mathrm{~cm}^{-1} ;{ }^{1} \mathrm{H}$ NMR $\left(\mathrm{CDCl}_{3}, 500 \mathrm{MHz}\right) \delta 1.03(\mathrm{~s}, 21 \mathrm{H}), 1.14(\mathrm{~d}, J=5.9 \mathrm{~Hz}, 3 \mathrm{H}), 1.24(\mathrm{bs}, 1 \mathrm{H})$, $1.53(\mathrm{~m}, 2 \mathrm{H}), 2.08(\mathrm{q}, J=7.5 \mathrm{~Hz}, 2 \mathrm{H}), 3.93$ (sextet, $J=5.9 \mathrm{~Hz}, 1 \mathrm{H}), 4.07(\mathrm{~d}, J=4.8 \mathrm{~Hz}, 2 \mathrm{H}), 5.66$ $(\mathrm{m}, 2 \mathrm{H}) ;{ }^{13} \mathrm{C} \mathrm{NMR}\left(\mathrm{CDCl}_{3}, 125 \mathrm{MHz}\right) \delta 12.52,18.15,23.43,27.99,39.22,63.84,68.02,128.84$, 133.39; Anal. Calcd for $\mathrm{C}_{16} \mathrm{H}_{34} \mathrm{O}_{2} \mathrm{Si}$; C, 67.07; H, 11.96. Found: C, 67.07; H, 12.23.

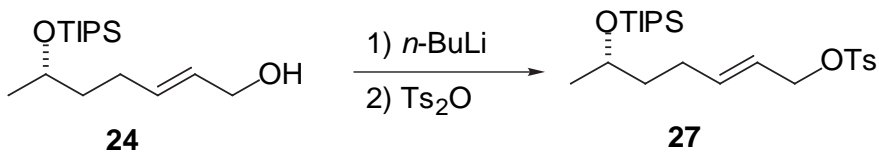

(S)-6-(triisopropylsiloxy)-hept-2-en-1-para-toluenesulfonate (27). $n$-BuLi (7.53 mL, 1.46 M, 1.05 equiv) was added dropwise to a solution of ( $S$ )-ethyl-6-(triisopropylsiloxy)-hept-2-en-1-ol $\left(\mathbf{2 4}, 3.0 \mathrm{~g}, 1.05 \times 10^{-2} \mathrm{~mol}, 1.0\right.$ equiv) in THF $(45 \mathrm{~mL})$ at $0{ }^{\circ} \mathrm{C}$. After 5 minutes, the solution was transfered via cannula to a solution of $p$-toluenesulfonic anhydride $\left(3.76 \mathrm{~g}, 1.16 \times 10^{-3} \mathrm{~mol}, 1.1\right.$ equiv) in THF ( $45 \mathrm{~mL})$ over a period of 10 minutes. After an additional 15 minutes of stirring, $\mathrm{CuI}$ (54 mg, $2.88 \times 10^{-3}$ mol, 0.1 equiv) was added while purging with nitrogen, and the solution was cannulated directly to the following reaction mixture without isolation.

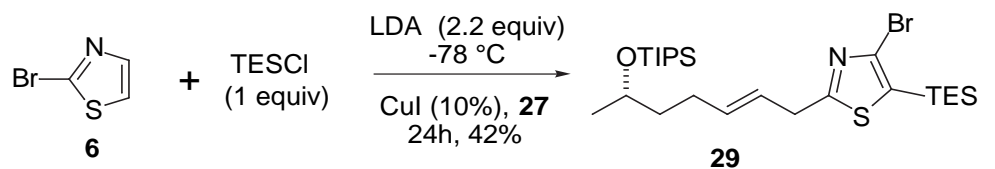

1-(4-Bromo-5-triethylsilyl-2-thiazolyl)-6-(S)-triisopropylsiloxy-hept-2-ene (29). $n$-BuLi (25.8 $\mathrm{mL}, 1.46 \mathrm{M}$ in hexanes, 3.6 equiv) was added dropwise to diisopropylamine $(5.87 \mathrm{~mL}, 4.2 \mathrm{x}$ $10^{-2}$ mol, 4.0 equiv) in THF $(100 \mathrm{~mL})$ at $0{ }^{\circ} \mathrm{C}$. After stirring for 15 minutes, the solution was cooled to $-78^{\circ} \mathrm{C}$. Triethylsilylchloride $\left(3.07 \mathrm{~mL}, 1.83 \times 10^{-3}\right.$ mol, 1.75 equiv) was added to the vigorously stirred LDA via syringe followed by 2-bromothiazole $\left(\mathbf{6}, 1.60 \mathrm{~mL}, 1.8 \times 10^{-2} \mathrm{~mol}, 1.7\right.$ equiv) via syringe over a period of 5 seconds. The light yellow solution was stirred for 2 hours at $-78{ }^{\circ} \mathrm{C}$. The $(S)$-6-(triisopropylsiloxy)-hept-2-en-1-tosylate (27) solution as prepared above was then transferred via cannula to the reaction mixture and stirred for 18 hours at $-78{ }^{\circ} \mathrm{C}$. The reaction was quenched with water at $-78{ }^{\circ} \mathrm{C}$, and allowed to come to room temperature. The reaction mixture was diluted with ethyl acetate, washed with $1 \mathrm{M} \mathrm{NH} 4 \mathrm{OH}$, saturated $\mathrm{NaHCO}_{3}$, and brine, dried over $\mathrm{MgSO}_{4}$, and concentrated under reduced pressure. Flash chromatography $\left(\mathrm{SiO}_{2}, 100 / 1\right.$ hexanes/diethyl ether) provided 1-(4-bromo-5-triethylsilyl-2-thiazolyl)-6-( $S$ )-triisopropylsiloxyhept-2-ene (29) (2.41 g, 42\% yield from $(S)$-ethyl-6-(triisopropylsiloxy)-hept-2-enol, 24). Rf 0.4 (50:1 Hexanes/Diethyl ether); $[\alpha]_{\mathrm{D}}+3.11^{\circ}$ (c $11.9 \mathrm{mg} / \mathrm{mL}, \mathrm{CH}_{2} \mathrm{Cl}_{2}$ ); IR (neat): 2956, 1465, 1016 $\mathrm{cm}^{-1} ;{ }^{1} \mathrm{H} \mathrm{NMR}\left(\mathrm{CDCl}_{3}, 500 \mathrm{MHz}\right) \delta 0.88(\mathrm{~m}, 6 \mathrm{H}), 0.96(\mathrm{~m}, 9 \mathrm{H}), 1.04(\mathrm{~s}, 21 \mathrm{H}), 1.15(\mathrm{~d}, J=5.9$ $\mathrm{Hz}, 3 \mathrm{H}), 1.53(\mathrm{~m}, 2 \mathrm{H}), 2.11(\mathrm{~m}, 2 \mathrm{H}), 3.67(\mathrm{~d}, J=6.7 \mathrm{~Hz}, 2 \mathrm{H}), 3.94($ sextet $, J=5.9 \mathrm{~Hz}, 1 \mathrm{H}), 6.64$ $(\mathrm{m}, 2 \mathrm{H}) ; 13 \mathrm{C} \mathrm{NMR}\left(\mathrm{CDCl}_{3}, 125 \mathrm{MHz}\right) \delta 3.72,7.28,12.50,18.15,23.39,28.24,36.62,39.16$, 67.97, 124.38, 125.26, 130.48, 135.53, 175.61; Anal. Calcd for $\mathrm{C}_{25} \mathrm{H}_{48} \mathrm{BrNOSSi}_{2}$; C, 54.91; $\mathrm{H}$, 8.85; N, 2.56; S, 5.86. Found: C, 54.86; H, 8.73; N, 2.54; S, 5.75.

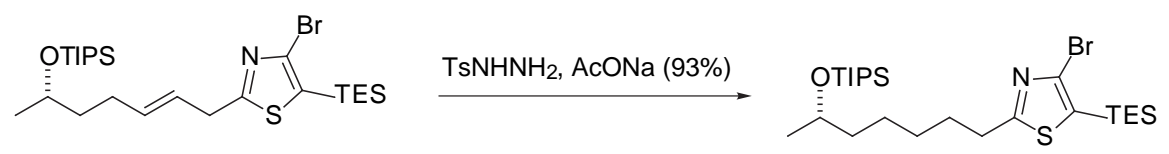

29

1-(4-Bromo-5-triethylsilyl-2-thiazolyl)-6- $(S)$-triisopropylsiloxy-heptane. 1-(4-Bromo-5triethylsilyl-2-thiazolyl)-6-(S)-triisopropylsiloxy-hept-2-ene (29) (1.50 g, 2.74 x 10-3 mol, 1.0 equiv) was placed in a round bottom flask with $p$-toluenesulfonohydrazine $\left(5.11 \mathrm{~g}, 2.74 \times 10^{-2} \mathrm{~mol}\right.$, 10 equiv) and DME (50 mL). The solution was brought to reflux, then sodium acetate $(7.46 \mathrm{~g}, 5.5$ x $10^{-2}$ mol, 20 equiv) in water $(30 \mathrm{~mL})$, was added dropwise over a period of 6 hours via cannula. The solution was heated to reflux for 18 hours, cooled, and diluted with ethyl acetate $(50 \mathrm{~mL})$. The 
organic layer was washed with saturated $\mathrm{NaHCO}_{3}$, and brine, dried over $\mathrm{MgSO}_{4}$, and concentrated under reduced pressure. Flash chromatography $\left(\mathrm{SiO}_{2}, 100 / 1\right.$ hexanes/diethyl ether) provided 1-(4Bromo-5-triethylsilyl-2-thiazolyl)-6-(S)-triisopropylsiloxy-heptane (1.40 g, 93\%). Rf 0.4 (50:1 hexanes/diethyl ether); $[\alpha]_{\mathrm{D}}+1.73^{\circ}\left(\mathrm{c} 11.0 \mathrm{mg} / \mathrm{mL}, \mathrm{CH}_{2} \mathrm{Cl}_{2}\right)$; IR (neat): $2940,1463,1017 \mathrm{~cm}^{-1}$; $1_{\mathrm{H} \mathrm{NMR}}\left(\mathrm{CDCl}_{3}, 500 \mathrm{MHz}\right) \delta 0.89(\mathrm{~m}, 6 \mathrm{H}), 0.96(\mathrm{~m}, 9 \mathrm{H}), 1.03(\mathrm{~s}, 21 \mathrm{H}), 1.12(\mathrm{~d}, J=6.1 \mathrm{~Hz}$, $3 \mathrm{H}), 1.40(\mathrm{~m}, 6 \mathrm{H}), 1.76(\mathrm{~m}, 2 \mathrm{H}), 2.96(\mathrm{t}, J=7.8 \mathrm{~Hz}, 2 \mathrm{H}), 3.89$ (sextet, $J=5.9 \mathrm{~Hz}, 1 \mathrm{H}) ; 13 \mathrm{C} \mathrm{NMR}$ $\left(\mathrm{CDCl}_{3}, 125 \mathrm{MHz}\right) \delta 3.74,7.28,12.49,18.14,23.49,24.90,29.36,29.83,33.30,39.71,68.43$, 124.51, 130.28, 176.43; Anal. Calcd for $\mathrm{C}_{25} \mathrm{H}_{50}$ BrNOSSi$_{2} ; \mathrm{C}, 54.71 ; \mathrm{H}, 9.18 ; \mathrm{N}, 2.55 ; \mathrm{S}, 5.84$. Found: C, 54.89; H, 9.08; N, 2.39; S, 6.19.
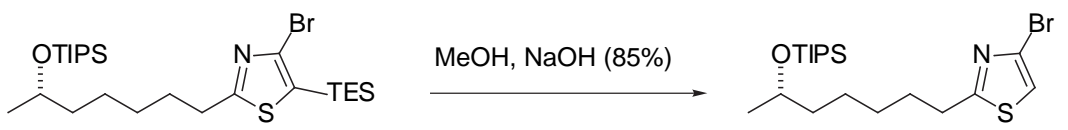

30

1-(4-Bromo-2-thiazolyl)-6-(S)-triisopropylsiloxy-heptane. 1-(4-Bromo-5-triethylsilyl-2thiazolyl)-6-(S)-triisopropylsiloxy-heptane (1.33 g, $2.42 \times 10^{-3} \mathrm{~mol}, 1.0$ equiv) was dissolved in 20 $\mathrm{mL}$ methanol and $10 \mathrm{M} \mathrm{NaOH}\left(2.4 \mathrm{~mL}, 2.4 \times 10^{-2}\right.$ mol, 10 equiv) and stirred for 18 hours at room temperature. The reaction was diluted with $25 \mathrm{~mL}$ ethyl acetate and washed with saturated $\mathrm{NaHCO}_{3}$, and brine, dried over $\mathrm{MgSO}_{4}$, and concentrated under reduced pressure. Flash chromatography $\left(\mathrm{SiO}_{2}, 100 / 1\right.$ hexanes/diethyl ether) provided 1-(4-Bromo-2-thiazolyl)-6- $(S)$ triisopropylsiloxy-hept-2-ene (1.01 g, 85\%). Rf 0.4 (50:1 hexanes/diethyl Ether); $[\alpha]_{\mathrm{D}}-1.09^{\circ}$ (c $12.9 \mathrm{mg} / \mathrm{mL}, \mathrm{CH}_{2} \mathrm{Cl}_{2}$ ); IR (neat): $2940,1482,1050,884 \mathrm{~cm}^{-1} ;{ }^{1} \mathrm{H} \mathrm{NMR}\left(\mathrm{CDCl}_{3}, 500 \mathrm{MHz}\right) \delta$ $1.03(\mathrm{~s}, 21 \mathrm{H}), 1.12(\mathrm{~d}, J=6.1 \mathrm{~Hz}, 3 \mathrm{H}), 1.40(\mathrm{~m}, 6 \mathrm{H}), 1.77(\mathrm{~m}, 2 \mathrm{H}), 2.97(\mathrm{t}, J=7.5 \mathrm{~Hz}, 2 \mathrm{H}), 3.89$ (sextet, $J=6.2 \mathrm{~Hz}, 1 \mathrm{H}), 7.05(\mathrm{~s}, 1 \mathrm{H}) ;{ }^{3}{ }^{3} \mathrm{C} \mathrm{NMR}\left(\mathrm{CDCl}_{3}, 125 \mathrm{MHz}\right) \delta 12.49,18.15,23.50,24.89$, 29.23, 29.84, 33.56, 39.70, 68.41, 115.62, 124.11, 172.73; Anal. Calcd for $\mathrm{C}_{25} \mathrm{H}_{50} \mathrm{BrNOSSi}_{2}$; C, 52.51; H, 8.35; N, 3.22; S, 3.68. Found: C, 52.47; H, 8.22; N, 3.05; S, 7.69.

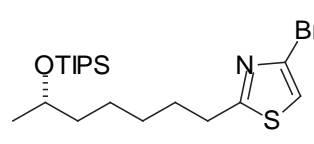

30

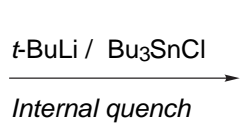

Internal quench

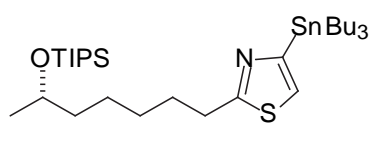

31

1-(4-Tributyltin-2-thiazolyl)-6-(S)-triisopropylsiloxy-heptane. $t$-BuLi $(981 \mu \mathrm{L}, 1.7 \mathrm{M}$ in pentane, 1.1 equiv) was added dropwise to a solution of 1-(4-Bromo-2-thiazolyl)-6- $(S)$ triisopropylsiloxy-heptane (30,659 mg, $1.52 \times 10^{-3} \mathrm{~mol}, 1.0$ equiv) and tributyltin chloride (354 $\mu \mathrm{L}, 2.13 \times 10^{-3} \mathrm{~mol}, 1.4$ equiv) in THF $(15 \mathrm{~mL})$ at $-78^{\circ} \mathrm{C}$. The solution was stirred for 1 hour at $-78{ }^{\circ} \mathrm{C}$, and then allowed to come to room temperature. The reaction was diluted with ethyl acetate $(15 \mathrm{~mL})$ and stirred for 1 hour with saturated aqueous $\mathrm{KF}(3 \mathrm{~mL})$. The organic layer was washed with saturated $\mathrm{NaHCO}_{3}$, and brine, dried over $\mathrm{MgSO}_{4}$, and concentrated under reduced pressure to provide $843 \mathrm{mg}$ of crude 1-(4-tributyltin-2-thiazolyl)-6-(S)-triisopropylsiloxy-heptane (31) which was used without purification (attempted flash chromatography resulted in significant amounts of destannylation) in the following reaction.

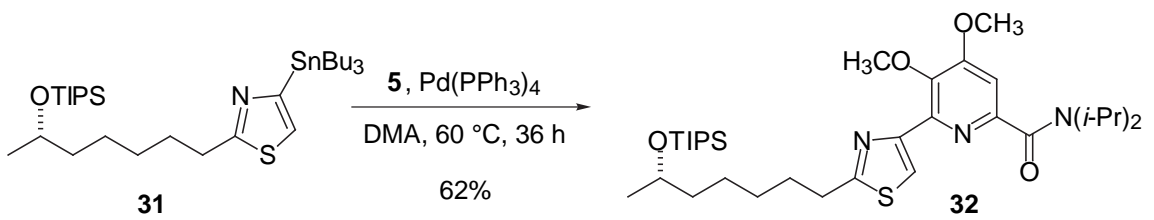

6-(2-(6-(S)-Triisopropylsiloxy-heptane)-4-thiazolyl)-4,5-dimethoxy-2-pyridine Carboxylic Acid $N, N$-Diisopropylamide (32). A solution of crude 1-(4-tributyltin-2thiazolyl)-6-(S)-triisopropylsiloxy-heptane $(\mathbf{3 1}, 843 \mathrm{mg})$ and 6-iodo-4,5-dimethoxy-2-pyridine carboxylic acid $N, N$-diisopropylamide $(5)^{3}\left(535 \mathrm{mg}, 1.37 \times 10^{-3}\right.$ mol, 0.9 equiv) in dimethylacetamide (DMA) (15 mL) was added to tetrakistriphenylphosphine palladium (175 mg, $1.52 \times 10^{-4} \mathrm{~mol}, 0.1$ equiv), CuI (29 mg, $1.52 \times 10^{-4} \mathrm{~mol}, 0.1$ equiv), and $\mathrm{LiCl}$ (128 mg, $3.04 \times 10^{-}$ 
3 mol, 2.0 equiv) under nitrogen. The solution was brought to $70{ }^{\circ} \mathrm{C}$ for 24 hours in an oil bath. Upon cooling, the DMA was distilled off under reduced pressure. The residue was dissolved in ethyl acetate $(10 \mathrm{~mL})$, and stirred for 1 hour with saturateed aqueous $\mathrm{KF}(15 \mathrm{~mL})$. The organic layer was washed with saturated $\mathrm{NaHCO}_{3}$, and brine, dried over $\mathrm{MgSO}_{4}$, and concentrated under reduced pressure. Flash chromatography $\left(\mathrm{SiO}_{2}, 9 / 1\right.$ chloroform/ethyl acetate) provided 6-(2-(6$(S)$-triisopropylsiloxy-heptane)-4-thiazolyl)-4,5-dimethoxy-2-pyridine carboxylic acid $N, N$ diisopropylamide $\left(32,447 \mathrm{mg}, 6 \times 10^{-4} \mathrm{~mol}, 53 \%\right)$. Rf $0.2\left(9 / 1\right.$ chloroform/ethyl acetate); $[\alpha]_{\mathrm{D}}$ $-1.58^{\circ}$ (c $13.9 \mathrm{mg} / \mathrm{mL}$, chloroform); ${ }^{1} \mathrm{H} \mathrm{NMR}\left(\mathrm{CDCl}_{3}, 500 \mathrm{MHz}\right) \delta 1.03(\mathrm{~s}, 21 \mathrm{H}), 1.13(\mathrm{~d}, J=6.1$ $\mathrm{Hz}, 3 \mathrm{H}), 1.23(\mathrm{~d}, J=6.5 \mathrm{~Hz}, 6 \mathrm{H}), 1.34-1.54(\mathrm{~m}, 6 \mathrm{H}), 1.53(\mathrm{~d}, J=6.7 \mathrm{~Hz}, 6 \mathrm{H}) 1.84(\mathrm{~m}, 2 \mathrm{H}), 3.04(\mathrm{t}$, $J=7.7 \mathrm{~Hz}, 2 \mathrm{H}), 3.55$ (septet, $J=6.7 \mathrm{~Hz}, 1 \mathrm{H}), 3.85(\mathrm{~s}, 3 \mathrm{H}), 3.55$ (sextet, $J=5.8 \mathrm{~Hz}, 1 \mathrm{H}), 3.95(\mathrm{~s}$, $3 \mathrm{H}), 4.30$ (septet, $J=6.5 \mathrm{~Hz}, 1 \mathrm{H}), 7.16(\mathrm{~s}, 1 \mathrm{H}), 7.81(\mathrm{~s}, 1 \mathrm{H}) ;{ }^{13} \mathrm{C} \mathrm{NMR}\left(\mathrm{CDCl}_{3}, 125 \mathrm{MHz}\right) \delta$ $12.49,18.15,20.60,20.94,23.52$, 25.06, 29.39, 29.80, 33.56, 39.79, 46.30, 50.78, 56.07, 60.89, $68.50,106.94,119.40,143.78,144.60,151.68,152.35,160.21,168.07,170.21$. Anal. Calcd for $\mathrm{C}_{33} \mathrm{H}_{57} \mathrm{~N}_{3} \mathrm{O}_{4} \mathrm{SSi}: \mathrm{C}, 63.93 ; \mathrm{H}, 9.27$; N, 6.78; S, 5.17. Found: C, 64.11; H, 9.44; N, 6.46; S, 5.11.

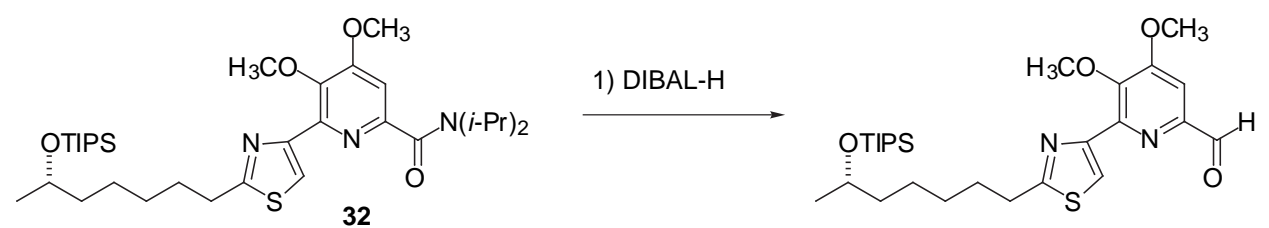

6-(2-(6-(S)-Triisopropylsiloxy-heptane)-4-thiazolyl)-4,5-dimethoxy-2-pyridine

Carboxaldehyde. Diisobutylaluminum hydride $(778 \mu \mathrm{L}, 1.0 \mathrm{M}$ in hexanes, 1.3 equiv) was added to a solution of 6-(2-(6-(S)-triisopropylsiloxy-heptane)-4-thiazolyl)-4,5-dimethoxy-2-pyridine carboxylic acid $N, N$-diisopropylamide $\left(32,371 \mathrm{mg}, 6.0 \times 10^{-4} \mathrm{~mol}, 1.0\right.$ equiv) in THF (10 mL) at 0 ${ }^{\circ} \mathrm{C}$, and then stirred for 1 hour. The reaction was diluted with hexanes $(3 \mathrm{~mL})$ and $\mathrm{NaOH}(600 \mu \mathrm{L}$, $10 \mathrm{M}$ ) was added dropwise by syringe. The reaction was allowed to stir at room temperature for 1 hour at which time a large amount of aluminum salts had precipitated. The reaction mixture was dried with $\mathrm{MgSO}_{4}$, filtered and concentrated under reduced pressure to provide 6-(2- $(6-(S)$ triisopropylsiloxy-heptane)-4-thiazolyl)-4,5-dimethoxy-2-pyridine carboxaldehyde (295 mg, 95\%) which was used without purification. ${ }^{1} \mathrm{H} \mathrm{NMR}\left(\mathrm{CDCl}_{3}, 500 \mathrm{MHz}\right) \delta 1.03(\mathrm{~s}, 21 \mathrm{H}), 1.13(\mathrm{~d}, J=$ $6.1 \mathrm{~Hz}, 3 \mathrm{H}), 1.37-1.52(\mathrm{~m}, 6 \mathrm{H}), 1.85(\mathrm{~m}, 2 \mathrm{H}), 3.13(\mathrm{t}, J=7.9 \mathrm{~Hz}, 2 \mathrm{H}), 3.90(\mathrm{~s}, 3 \mathrm{H}), 3.90(\mathrm{~m}, 1 \mathrm{H})$, $4.01(\mathrm{~s}, 3 \mathrm{H}), 7.53(\mathrm{~s}, 1 \mathrm{H}), 7.97(\mathrm{~s}, 1 \mathrm{H}), 10.09(\mathrm{~s}, 1 \mathrm{H}) ;{ }^{13} \mathrm{C} \mathrm{NMR}\left(\mathrm{CDCl}_{3}, 125 \mathrm{MHz}\right) \delta 12.52$, $18.15,23.43,27.99,39.22,63.84,68.02,128.84,133.39$.

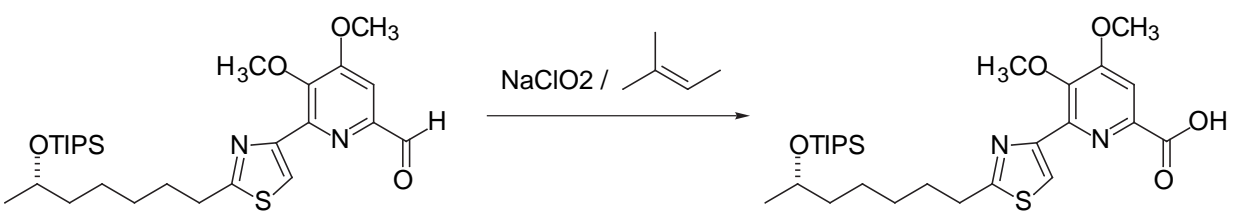

6-(2-(6-(S)-Triisopropylsiloxy-heptane)-4-thiazolyl)-4,5-dimethoxy-2-pyridine

Carboxylic Acid. 6-(2-(6-(S)-Triisopropylsiloxy-heptane)-4-thiazolyl)-4,5-dimethoxy-2-pyridine carboxaldehyde (167 mg, $3.2 \times 10^{-4}$ mol, 1.0 equiv), and $\mathrm{NaH}_{2} \mathrm{PO}_{4}\left(192 \mathrm{mg}, 1.6 \times 10^{-4} \mathrm{~mol}, 5.0\right.$ equiv) were dissolved in $t$ - $\mathrm{BuOH}(2 \mathrm{~mL})$, water $(2 \mathrm{~mL})$ and 2-methyl-2-butene $(4 \mathrm{~mL})$. Sodium chlorite (192 mg, $1.6 \times 10^{-4} \mathrm{~mol}, 5.0$ equiv) was added in three portions over a period of one minute. The reaction was allowed to stir at room temperature for 2 hours, and then diluted with ethyl acetate. The organic layer was washed with saturated $\mathrm{NaHCO}_{3}$, and brine, dried over $\mathrm{MgSO}_{4}$, and concentrated under reduced pressure. The crude product was used without purification in the following reaction. 


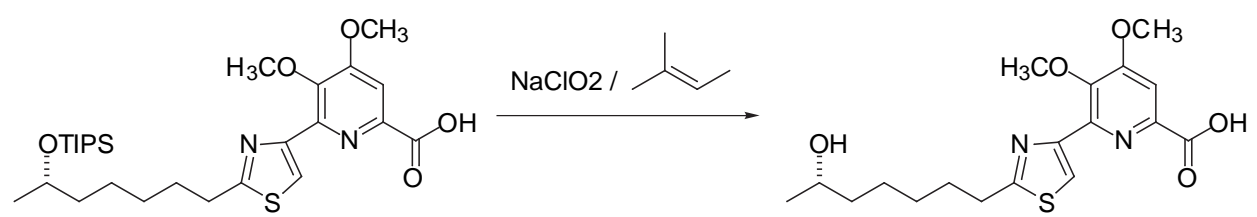

(+)-(S)-WS75624 B, 2b

(+)-(S)-WS75624 B (2b). 6-(2-(6- $(S)$-Triisopropylsiloxy-heptane)-4-thiazolyl)-4,5-dimethoxy2-pyridine carboxylic acid (3.2 x 10-4 mol, 1.0 equiv) and tetrabutylamonium flouride (251 mg, 9.6 $\mathrm{x} 10^{-4} \mathrm{~mol}, 3.0$ equiv) were dissolved in THF (5 mL) and allowed to stir at room temperature overnight. The reaction mixture was diluted with ethyl acetate, and the organic layer was washed with saturated $\mathrm{NaHCO}_{3}$, and brine, dried over $\mathrm{MgSO}_{4}$, and concentrated under reduced pressure. The resulting solid was then recrystallized from hot ethyl acetate to obtain $(+)-(S)$-WS75624B (2b) (85 mg, 70\% yield from 6-(2-(6-(S)-triisopropylsiloxy-heptane)-4-thiazolyl)-4,5-dimethoxy-2pyridine carboxaldehyde) $[\alpha]_{\mathrm{D}}+3.1^{\circ}\left(\mathrm{c} 8.0 \mathrm{mg} / \mathrm{mL}, \mathrm{CH}_{3} \mathrm{OH}\right) ;{ }^{1} \mathrm{H}$ NMR $\left(\mathrm{CD}_{3} \mathrm{OD}, 500 \mathrm{MHz}\right) \delta$ $1.13(\mathrm{~d}, J=6.2 \mathrm{~Hz}, 3 \mathrm{H}), 1.39-1.49(\mathrm{~m}, 6 \mathrm{H}), 1.86$ (pent, $J=7.5 \mathrm{~Hz}, 2 \mathrm{H}), 3.12(\mathrm{t}, J=7.6 \mathrm{~Hz}, 2 \mathrm{H})$, 3.70 (sextet, $J=6.3 \mathrm{~Hz}, 1 \mathrm{H}), 4.01(\mathrm{~s}, 3 \mathrm{H}), 4.10(\mathrm{~s}, 3 \mathrm{H}), 7.83(\mathrm{~s}, 1 \mathrm{H}), 8.35(\mathrm{~s}, 1 \mathrm{H})$; $13 \mathrm{C}$ NMR $\left(\mathrm{CD}_{3} \mathrm{OD}, 125 \mathrm{MHz}\right) \delta 23.52,26.53,30.21,31.34,34.00,39.98,57.44,61.02,68.44,109.17$, 123.83 142.89, 144.75, 147.36, 148.36, 163.37, 166.63, 173.55. Anal. Calcd for $\mathrm{C}_{18} \mathrm{H}_{24} \mathrm{~N}_{2} \mathrm{O}_{5} \mathrm{~S}$ C, 56.82; H, 6.36; N, 7.36; S, 8.43. Found: C, 56.46; H, 6.23; N, 7.18; S, 8.47. The spectral properties for $\mathbf{2 b}$ are identical to those reported in the literature. ${ }^{4}$

1 Perrin, D. D. ; Armarego, W. L. F. Purification of Laboratory Chemicals Pergamon: Oxford, 1988.

2 Ohkuma, T.; Kitamura, M.; Noyori, R. Tetrahedron Lett. 1990, 31, 5509.

3 For the synthesis of this compound, see: Sammakia, T.; Stangeland, E. L.; Whitcomb, M. C. Org. Lett. 2002; 4; 2385

4 Yoshimura, S.; Tsurumi, T.; Takase, S.; Okuhara, M. J. Antibiotics 1995, 48, 1073. 